\title{
1 Single Cell atlas of uterine myometrium and leiomyomas reveals diverse and novel
}

\section{2 cell types of non-monoclonal origin.}

3 Jyoti Goad ${ }^{1,2^{*}}$, Joshua Rudolph ${ }^{3}$, Jian-Jun Wei $^{4}$, Serdar E Bulun ${ }^{5}$, Debabrata Chakravarti ${ }^{5}$,

4 Aleksandar Rajkovic ${ }^{1,2,6^{*}}$

$5 \quad{ }^{1}$ Department of Pathology, University of California, San Francisco, CA, 94143, USA

$6{ }^{2}$ Department of Obstetrics, Gynecology and Reproductive Sciences, University of California,

7 San Francisco, CA, 94143, USA

$8{ }^{3}$ Lung Biology Center, Department of Medicine, University of California, San Francisco, USA

$9{ }^{4}$ Department of Pathology, Northwestern University, Chicago, IL, 60611, USA

$10{ }^{5}$ Department of Obstetrics and Gynecology, Division of Reproductive Science in Medicine,

11 Feinberg School of Medicine, Northwestern University, Chicago, IL, 60611, USA

$12{ }^{6}$ Institute of Human Genetics, University of California, San Francisco, CA, 94143, USA,

*Address correspondence to:

Dr. Aleksandar Rajkovic

HSE-901, 513 Parnassus Ave

San Francisco, California

USA-94143

Ph: 415-502-4961

Email: aleks.rajkovic@ucsf.edu

Or

Dr. Jyoti Goad

HSE-901, 513 Parnassus Ave

San Francisco, California

USA-94143

Ph: 415-514-4687

Email: Jyoti.goad@ucsf.edu 
35 Keywords: Uterine leiomyomas, single cell sequencing, MED12, monoclonal

\section{Abstract}

37 Uterine leiomyomas are the most common tumors of the female reproductive tract with 38 significant morbidity that includes excessive bleeding, infertility and pregnancy 39 complications. The origin and cellular composition of leiomyomas is controversial, yet very 40 important in better understanding the pathogenesis of these tumors. We applied single-cell

41 RNA sequencing to better understand cellular heterogeneity of uterine leiomyomas and 42 normal myometrium at the molecular level. Our data reveal previously unknown 43 heterogeneity in the smooth muscle cells, fibroblast cells, and endothelial cells of normal 44 myometrium and leiomyomas. We discovered a novel lymphatic endothelial cell population 45 in uterine leiomyomas and that the immune as well as transcriptional profile of leiomyomas 46 is MED12 genotype-dependent. Moreover, we show that leiomyoma cell moiety is not 47 monoclonal in nature. Our work describes unprecedented single cell resolution of normal 48 uterine myometrium and leiomyoma tumors and provides insight into tumor specific hormone responsiveness and extracellular matrix accumulation.

\section{Introduction}

Uterine leiomyomas, also known as fibroids, are benign tumors of the myometrium

53 affecting nearly $25 \%$ of women in their reproductive age ${ }^{1}$. These tumors significantly affect 54 women's quality of life and are the single most common cause of hysterectomy ${ }^{1}$. Each year, approximately 300,000 myomectomies and 200,000 hysterectomies are performed in the

56 United States to remove either leiomyoma tumors or the whole uterus ${ }^{2,3}$. Despite its 57 importance to women's health, there are currently no leiomyoma specific therapeutics. 58 Moreover, the underlying origin and heterogeneity of leiomyomas continues to evolve. Extensive genetic studies from our group and others have shown MED12 exon 2 as

60 a hotspot for genetic variants that associate with leiomyoma in about $70 \%$ of the cases ${ }^{4-6}$. In 61 contrast, the MED12 variant negative leiomyomas have a highly heterogenous genomic 62 landscape $\mathrm{e}^{7,8}$. Studies have shown that MED12 variant negative leiomyomas are bigger than 
63 leiomyomas expressing MED12 variant allele ${ }^{9}$. The genotype dependent difference in

64 leiomyoma size is due to differences in the cell composition, rate of proliferation, and

65 accumulation of extracellular matrix ${ }^{10}$. Leiomyomas are considered monoclonal tumors of

66 smooth muscle cell in uterus ${ }^{11}$. However, recent histological and flow cytometry studies have

67 shown the presence of smooth muscle cells, fibroblasts, endothelial cells and immune cells

68 in leiomyomas ${ }^{12}$. How these cells contribute to the leiomyoma formation and the molecular

69 salient characteristics of the cellular heterogeneity in leiomyomas remain undetermined.

$70 \quad$ Here we utilized single-cell RNA sequencing to understand the underlying cellular

71 heterogeneity in normal myometrium and uterine leiomyomas. We have identified two novel

72 lymphatic endothelial cell populations that are present in uterine leiomyomas. We also

73 determined the transcriptomic changes in the leiomyoma cell clusters based on the genetic

74 mutation. Our data shows that different smooth muscle and fibroblast cell clusters expand in

75 leiomyomas as compared to myometrium. Immune cell infiltration differs with the genotype

76 of the leiomyomas. Our work has identified unanticipated cellular differences in leiomyomas

77 and the myometrium, which could help the future direction of developing targeted therapy for

78 leiomyoma treatment.

\section{Methods}

\section{Study subjects}

82 To resolve the cellular identity of the cells, we collected 66,339 cells from the leiomyomas

83 and the myometrium from a total of 8 patients. Five out of the 8 samples were matched 84 which were collected from patients undergoing hysterectomy for the treatment of 85 leiomyomas. The non-matched leiomyomas were collected from patients undergoing 86 myomectomy. Histopathological assessment of the collected samples confirmed the identity 87 of the samples.

\section{Patient tissue collection and genotyping}


90 Uterine leiomyomas and normal myometrium were collected from the patients undergoing

91 hysterectomy or myomectomy with informed consent. The study was approved by the UCSF

92 institutional review board, ethics approval 17-22669. Fresh tissue samples were collected

93 immediately after the surgery, placed in ice cold DMEM/F12, and immediately sent to the

94 lab. A small part of each tissue obtained was snap frozen to perform DNA isolation for

95 genotyping as described previously by us $^{4}$. Additionally, a part of the sample was fixed in

96 formalin overnight for histology. Patient information is provided in S table 1.

\section{Preparation of single cell suspension from the fresh tissues}

99 The tissue samples were collected and washed in HBSS (Sigma). Leiomyomas and 100 myometrium were cut into pieces of 3-4 mm. These pieces were then added to $3-4 \mathrm{ml}$ of 101 digestion media containing $0.1 \mathrm{mg} / \mathrm{ml}$ Liberase (Roche, 501003280), $100 \mathrm{U} / \mathrm{ml}$ DNase I 102 (Sigma, D4527), and $25 \mathrm{U} / \mathrm{ml}$ Dispase (Sigma, D4818) in DMEM (Life Technologies, 12634010) per gram of the tissue and mechanically dissociated using gentle MACS dissociator (Miltenyi Biotech, Germany) for 30 mins at $37^{\circ} \mathrm{C}$ to prepare a single cell suspension. The cell suspension was then pipetted up and down with $25 \mathrm{ml}, 10 \mathrm{ml}$, and $5 \mathrm{ml}$ pipettes for 1 minute each and then filtered through $70 \mu \mathrm{m}$ filter (Corning, 431751). Debris

107 was then removed from the cell suspension using debris removal solution (Miltenyi Biotech, 108 130-109-398) as per the manufacturer's instructions. The cells were then incubated with 109 RBC lysis buffer (Thermofisher Scientific, 00-4333-57) for 5 mins on ice to remove the red

110 blood cells. The cells were then resuspended in PBS containing $0.4 \%$ ultrapure BSA

111 (Thermofisher Scientific, AM2616) and passed through the 70- $\mu \mathrm{m}$ cell strainer (Bel-Art,

$112 \mathrm{H} 13680-0070)$ to obtain single cell suspension.

\section{Immunofluorescence and RNAscope}

114 Immunofluorescence was performed as described previously ${ }^{13}$. Briefly, tissue sections were 115 incubated with the following primary antibodies: CD8a (1:200, Cell signaling), CD20 (1:250, 
116 Abcam), NUSAP1 (1:1000, Abcam), PDPN (1:200, Cell signaling), overnight at $4^{\circ} \mathrm{C}$, then

117 incubated with secondary antibody for 1 hour at room temperature. For

118 immunofluorescence, the images for all of the samples; were taken at the same exposure

119 using a Nikon microscope (Nikon, Japan).

120 RNAScope Fluorescent multiplex assay was performed using the RNAScope Multiplex

121 Fluorescent v2 kit (Advanced Cell Diagnostics) as per the manufacturer's instructions.

122 Images were obtained using the Leica SP8 confocal microscope (Leica Biosystems,

123 Germany).

\section{Dual immunofluorescence and in-situ hybridization}

125 Dual immunofluorescence and in-situ hybridization were performed using the RNAScope

126 Multiplex Fluorescent Reagent kit v2 (Advanced Cell Diagnostics) as per the manufacturer's

127 protocol. Briefly, after the RNAScope protocol, the tissue sections were blocked overnight at

$1284^{\circ} \mathrm{C}$. Tissues were then incubated with $\alpha$-sma (1:200, Sigma Aldrich) antibody for an hour at

129 room temperature followed by incubation with the secondary antibody for $1 \mathrm{hr}$ at room

130 temperature. Images were obtained using the Leica SP8 confocal microscope (Leica

131 Biosystems, Germany).

\section{Single cell sequencing}

133 Single cells were processed through the 10X Chromium system (10X Genomics, USA) using

134 single cell 5' library and Gel bead kit (10X Genomics) as per the manufacturer's instructions.

135 Briefly, the single cell suspensions were partitioned into gel bead-in-emulsions which were

136 utilized to generate the barcoded cDNA libraries. The single-cell barcoded cDNA libraries

137 were then sequenced using an Illumina NovaSeq 6000 sequencing system (Illumina, USA).

\section{Pre-processing scRNA-seq data}


139 Cellranger v.2.1.0 single-cell software suite from 10X Genomics was used to demultiplex

140 fastq files, align reads to the Genome Reference Consortium Human Build 38 (hg38)

141 transcriptome, and extract cell and UMI barcodes. Raw cell count by transcript matrices

142 were imported into $\mathrm{R}$ and Seurat for analysis ${ }^{14}$. Each sample's expression matrix was filtered

143 to remove low-quality cells, defined as having fewer than 200 reads, greater than 2500

144 reads, or more than seven percent mitochondrial gene expression. Samples were merged

145 using Seurat's integration anchors. Any cells with more than one percent hemoglobin gene

146 expression were removed, with hemoglobin genes considered as $H B A 2, H B A 1$, and $H B B$.

147 The remaining hemoglobin gene expression was regressed out from the expression matrix

148 using Seurat's scale.data feature. To counteract differences in sequencing depth across

149 cells, transcript counts were normalized in each cell to transcripts per 10,000 unique

150 molecular identifiers (UMI).

151 Dimensionality reduction, clustering, and differential expression analysis

152 Seurat was used to cluster the merged object into subsets of cells. This workflow includes

153 finding variable genes, running principal component analysis on variable genes, running

154 Uniform Manifold Approximation and Projection on Principal components (UMAP) (1:20),

155 and graphed clustering using KNN and Louvain clustering (with Seurat FindClusters ()

156 resolution 0.4). Cell types were defined empirically using expression of marker genes. Cells

157 were partitioned into smooth muscle, fibroblasts, endothelial, and immune cells based on the

158 known signature markers. The following signature markers were used to identify the cell

159 clusters: smooth muscle cells (MYH11, TAGLN, ACTA2, CNN1, DES, CALD1), fibroblast

160 cells (VIM, ALDH1, CD90, FN1, DCN, OGN, MGP, COL1A1, COL1A2, COL3A1),

161 endothelial cells (PECAM1, CD31, CDH11, VWF), and immune cells (CD3D, CD3E,

162 FCER1G, MS4A1, CD79B, CST7, GZMB, FCGF3A, MS4A7).

163 Further clustering was performed for each cell type based on the expression of the signature

164 markers (with resolution 0.4 ) to delineate heterogeneity. For all clusters heatmaps, $t$ - 
165 distributed stochastic neighbor embedding ( $t$-SNE) and UMAP visualizations, violin plots and

166 dot plots were produced using Seurat functions with ggplot2, pheatmap and grid $\mathrm{R}$

167 packages. Differential gene expression analysis was performed using Seurat based on the 168 non-parametric Wilcoxon rank sum test.

\section{Batch effect and quality control}

170 Cell barcode and gene matrices were constructed using Cellranger and analyzed in R with

171 Seurat ${ }^{14}$. Cells with fewer than 200 features and genes in less than 3 cells were filtered. To

172 account for potential doublets and low-quality cells in the analysis, only cells with fewer than

1732500 features and less than 7 percent total mitochondrial genes were used for downstream

174 analyses. Cell samples were then combined using the Seurat standard workflow to integrate

175 cells. $H B A 2, H B A 1$, and $H B B$ genes were used to calculated hemoglobin gene percentage,

176 some cells with high percent were removed, and the remaining effect was regressed out

177 using the Scale.Data feature. Erythrocytes were also removed from downstream analysis.

\section{Standard Error Barplots}

179 Percentages of samples were used instead of raw cell numbers to normalize for the varying 180 number of cells introduced to the experiment by each sample. For each subset of cells, cell proportions were calculated as a percentage of the number of cells in a cluster from a sample, divided by total number of cells from the same sample. Standard error of the mean was then calculated for each cluster of samples and median percentages per cluster were used to plot.

Volcano Plots

187 Seurat's FindMarkers with default parameters was used to get the fold change between 188 MED12 positive leiomyomas and myometrium as well as MED12 negative leiomyomas and myometrium. All data was compiled and plotted in R with ggplot. 


\section{Clonality analysis}

192 Dsc-pileup in the freemuxlet tool was used to construct barcode and variants table ${ }^{15,16}$.

193 Variants from cells which weren't filtered by preprocessing were used in conjunction with

194 Seurat meta data to print UMAPs with variant information.

\section{Statistical Analysis}

196 Dirichlet multinomial regression was utilized to determine the statistical confidence that

197 proportions of conditions in each cluster are not a product of random sampling. A Fisher t-

198 test was also leveraged to compare cell numbers from one condition to another in each

199 cluster. Bar plots show means of sample percentages, with standard error of the mean 200 confidence intervals.

201

202

\section{RESULTS}

\section{Single cell atlas of the human myometrium and leiomyomas}

204 We collected a total of 66,339 cells present in normal myometrium and leiomyomas from a

205 total of eight patients (Fig. 1A). After accounting for technical and biological variation, 206 clustering of the 34,435 high quality cells $(11,235$ from myometrium $(n=5), 15,417$

207 from MED12 variant positive $(n=5)$ and, 7,783 cells from MED12 variant negative

208 leiomyomas $(n=3)$ ) revealed the presence of 18 different clusters across known cell lineages

209 (Fig. 1B, C). These cell clusters were highly reproducible as all samples were represented in

210 nearly all clusters (Fig. 1D). The subpopulations included known cell types previously

211 identified through histology and flow cytometry ${ }^{12}$. These included: smooth muscle cells,

212 fibroblasts, NK cell, T cells, B cells, myeloid cells, and endothelial cells, (Fig. 1C). The

213 annotations were performed using the differential gene expression analysis supported by

214 known gene markers such as ACTA2, CNN1 for smooth muscle cells (SMC), VWF and

215 PECAM for endothelial cells (Endo), PDPN lymphatic endothelial cells, DCN and LUM for

216 fibroblasts, CD3D for T cells, MS4A1 and CD79A for B cells, GNLY and NKG7 for NK cells, 
217 CD14, and S100A8 for myeloid cells, (Fig. 1E, S Fig. 1B,C). All cell clusters were present in

218 most patient samples across all three types of tissue samples: myometrium, MED12 variant

219 positive leiomyomas and MED12 variant negative leiomyomas (Fig. 1D, S Fig. 1A). The

220 contaminating endometrial cells (EPCAM+ or KRT8+) and red blood cells expressing HBB-

221 A/ HBA-2 were removed from the datasets from further analysis. Quality control matrices

222 were highly reproducible across patient samples and conditions (S Fig. 1D).

224 Characterization of the smooth muscle cells and fibroblast populations in normal myometrium

226 The cellular composition and molecular characterization of the normal myometrium are 227 poorly understood. To examine the cellular heterogeneity in the myometrium, we first 228 generated a UMAP from the myometrium dataset only. We identified the presence of 18 229 different cell clusters. These clusters included smooth muscle cells, fibroblast cells, 230 endothelial cells, lymphatic endothelial cells, and immune cell populations (S Fig.2A).

231 To determine if there is an additional underlying heterogeneity in the SMC population 232 of the normal myometrium, we extracted all the cells expressing TAGLN, CNN1 and SMA, 233 and clustered them at a higher resolution. These genes are known markers of SMC in uterus 234 and other tissues ${ }^{17,18}$. Additional re-clustering of these cells revealed five different smooth 235 muscle cell clusters in the normal myometrium (S Fig. 2B,F, Fig. 2E). All of the clusters were 236 present in all patient samples, indicating reproducibility and absence of technical or batch 237 effects (S Fig. 2C).

238 To delineate the functional role of the SMC, we performed gene ontology (GO) 239 enrichment analysis. Functional enrichment analysis with the biological function revealed 240 that SMC Cluster 0 was specifically associated with intracellular sequestering of iron ion, 241 cellular homeostasis, and response to stimulus. SMC Cluster 1 was defined by unique 242 expression of the gene CACNA1C and was associated with muscle contraction, collagen 243 fibril organization, extracellular matrix assembly and cellular response to TGF- $\beta$. SMC 244 Cluster 2 was enriched in HSPB6 and MFAP4. These cells were associated with 
245 glucocorticoid regulation, extracellular matrix (ECM) assembly and organization. SMC

246 Cluster 3 was defined by the unique expression of genes RGS5 and HIGD1B, which are

247 specifically responsible for regulating the cellular response to TNF, regulation of endothelial

248 cell proliferation. SMC Cluster 4 was enriched in the expression of TBX2, PDGFRB, ELN,

$249 \mathrm{NOTCH} 3$ and MCAM which are responsible for transcription by RNA polymerase II and

250 showed genes upregulated for stem cell differentiation. A full list of the GO processes for all

251 of the clusters is provided in the supplementary table (S Table 2). We performed in situ

252 hybridization to validate the biological presence of these clusters, using the markers

253 aSMA ${ }^{+}$, CACNA1C $^{-}, \mathrm{HSPB6}^{-}, \mathrm{RGS5}^{-}, \mathrm{TBX}^{-}$(SMC Cluster 0), CACNA1C (SMC Cluster 1),

254 HSPB6 (SMC Cluster 2), RGS5 (SMC Cluster 3), and TBX2 (SMC Cluster 4) (Fig. 2E).

255 Our data analysis also identified two distinct cell subsets within fibroblast cell

256 populations (S Fig. 2D,E). These fibroblasts differ from each other in the expression of

257 SFRP2, PLAC9, SERPINF1, S100A10 (S Fig. 2G). In situ hybridization confirmed the

258 presence of two different fibroblast cell populations (Fig. 3E) We next investigated the

259 biological function of these fibroblasts by performing gene ontology (GO) enrichment

260 analysis. We found that the WNT regulated cluster (Fibro Cluster 0 ) was mainly involved in

261 regulating fibroblast proliferation, ECM constituent secretion, and wound healing (S Table 3).

262 Fibro Cluster 1 was linked to cellular component organization or biogenesis and regulation of

263 mRNA splicing (S Table 3).

265 Distinct smooth muscle cell populations and fibroblast populations expand in 266 leiomyomas

267 To identify the differences in the cell composition between leiomyomas and normal 268 myometrium, we generated a UMAP for the leiomyoma-only dataset. We identified the 269 presence of same cell clusters as identified in the normal myometrium which were 270 constituted of smooth muscle cells, fibroblast cells, endothelial cells, T-cells, B-cells, NK 271 cells, and myeloid cell populations (S Fig. 3A,B). 
273 single mutant smooth muscle stem cell ${ }^{11}$. However, our data shows the presence of multiple

274 cell types in leiomyomas which are similar to the normal myometrium (SFig. 2, 3). To

275 understand if there is further intracellular heterogeneity in leiomyomas, and whether this

276 heterogeneity is genotype dependent, we categorized leiomyoma samples as MED12

277 variant positive and MED12 variant negative based on the presence on MED12 variant.

278 Then, we isolated the smooth muscle, fibroblast, endothelial cells and immune cell

279 populations from the integrated dataset and resolved them at higher resolution. Our data

280 analysis revealed the presence of five different smooth muscle cell populations in both

281 MED12 variant positive and MED12 variant negative leiomyomas (Fig. 2A-C). All of these

282 smooth muscle cell clusters were also present in normal myometrium (SFig. 2A). However,

283 we observed CACNA1C smooth muscle cell expansion (SMC Cluster 1) in MED12 variant

284 positive leiomyomas by 3-fold in comparison to the normal myometrium and MED12 variant

285 negative leiomyomas ( $P$ value $=0.04$, three-fold change) (Fig. 2D-G). Whereas in MED12

286 variant negative leiomyomas, SMC Cluster $2(H S P B 6+)$ expands compared to the normal

287 myometrium ( $P$ value $=0.1$, three-fold change Fig. $2 \mathrm{D}-\mathrm{G}$ ).

288 Next, we wanted to determine if similar heterogeneity exists in the fibroblast cell 289 population in leiomyomas. We found presence of two distinct fibroblast subsets in all the 290 three tissue samples, myometrium, MED12 variant positive, and MED12 variant negative 291 leiomyomas (Fig. 3A-C). We observed a significant expansion of Fibro Cluster 0 in both 292 MED12 variant positive ( $P$ value $=0.02,1.14$-fold change $)$ and MED12 variant negative $(P$ 293 value $=0.1,1.14$-fold change) leiomyomas compared to normal myometrium (Fig. 3D). This 294 cluster seems to be regulated by the WNT signaling pathway (Fig. 3C). In situ hybridization 295 for SFRP2 confirmed the expansion of the WNT regulated fibroblasts in the MED12 variant 296 positive and MED12 variant negative fibroblasts (Fig. 3E-J). Together, these datasets show 297 the presence of a heterogenous smooth muscle cell and fibroblast cell populations in both MED12 variant positive and MED12 variant negative leiomyomas. 
300 Remodeling of the smooth muscle and fibroblast cell populations in leiomyomas

301 compared to the myometrium

302 Our previous study has shown that mutation in MED12 is sufficient to cause leiomyoma 303 formation ${ }^{19}$. In this study we found that normal myometrium, and leiomyomas have similar 304 cellular composition (Fig. 1B-D, S Fig. 2A, S Fig. 3A). We hypothesized that transcriptomic 305 changes in normal myometrium due to the acquired genetic mutations are responsible for 306 the leiomyoma formation.

307 To determine that, we compared the transcriptomic changes in smooth muscle cell 308 subsets of MED12 variant positive leiomyomas, and MED12 variant negative leiomyomas to 309 the normal myometrium. Gene ontology analysis of the MED12 variant positive leiomyomas 310 revealed enrichment for genes involved in the regulation of fibroblast proliferation, smooth 311 muscle cell proliferation, inflammatory response, extracellular matrix organization, cellular 312 response to hormones and proliferation of activated T-cells. These genes were found across 313 the smooth muscle cell cluster in MED12 variant positive leiomyomas compared to 314 myometrium (Fig, 2H-K, S Table 4). We found significant expansion of the SMC Cluster 1 315 (CACNA1C) in MED12 variant positive leiomyomas. These cells are enriched across genes 316 regulating myoblast proliferation, response to muscle stretch, muscle cell differentiation, 317 regulation of fibroblast growth factor production, and establishment and maintenance of 318 cytoskeleton polarity (Fig. 2H,I, S table 4).

319 We found that SMC Cluster 2 was most enriched in the MED12 variant negative 320 leiomyomas in comparison to the normal myometrium (Fig 2D). Further analysis of the 321 transcriptomic changes in the smooth muscle cells of the MED12 variant negative 322 leiomyomas compared to the normal myometrium revealed that in SMC Cluster 2, genes 323 involved in the biological processes such as retinoid metabolic process (GPC3, STRA6, $324 A D H 1 B, C R A B P 2, H S P G 2, L R P 1)$, positive regulation of non-canonical $W N T$ signaling 325 pathway (GPC3, DAB2, SFRP1) and negative regulation of smooth muscle cell-matrix 326 adhesion (SERPINE1, APOD) were enriched (Fig 2J,K, S Table 4). The full list of other 327 transcriptomic changes in the smooth muscle clusters are provided in S table 4. 
MED12 variant positive and MED12 variant negative leiomyomas is due to differences in

330 their cellular composition. They have suggested that in MED12 variant positive leiomyomas

331 both smooth muscle cells and fibroblast cell populations secrete collagen ${ }^{10}$. So, we decided

332 to compare the transcriptional changes in MED12 variant positive and MED12 variant

333 negative leiomyomas' fibroblast subset to that of the myometrium. We found upregulation of

334 the Vitamin A metabolic process, retinoic acid biosynthesis process, cellular response to the

335 vascular endothelial growth response, collagen fibril formation and inflammatory response in

336 MED12 variant positive leiomyoma's Fibro Cluster 0 (Fig. 3K,M). This fibroblast population is

337 regulated by WNT signaling in normal myometrium and expands in the MED12 variant

338 positive leiomyomas (Fig. 3D). While MED12 variant positive Fibro Cluster 1 had genes

339 induced for chronic inflammatory response, wound healing, BMP signaling, ECM

340 organization and positive regulation of the transcription by RNA polymerase II (Fig. 3L,N).

341 Interestingly, similar to MED12 variant positive leiomyomas Fibro Cluster 0 also expands in

342 MED12 variant negative leiomyomas. While we observed significant transcriptional changes

343 in both fibroblast cell clusters 0 and 1 in MED12 variant positive leiomyomas as compared to

344 normal myometrium, only WNT regulated fibroblasts (Fibro Cluster 0 ) showed transcriptional

345 differences in MED12 variant negative leiomyomas compared to the normal myometrium

346 (Fig. 3L,N, Supplementary Table 5). We did not observe any significant transcriptional

347 differences in Fibro cluster 1 between normal myometrium and MED12 variant negative

348 leiomyomas (Fig. 3L,N). Collectively, these data suggest that smooth muscle cells and

349 fibroblast cells expand and remodel differently according to the leiomyoma's genotype.

\section{Novel lymphatic endothelial cells inhabit leiomyomas}

352 Unsupervised clustering of endothelial cells revealed the presence of nine clusters of 353 endothelial cells (Fig. 4A-C). We observed that Endo cluster 8 was exclusively expressed in 354 both MED12 positive and MED12 negative leiomyomas but not in normal myometrium (Fig. 355 4B,D). The Endo cluster 8 cells were present in all patient leiomyoma samples (S Fig. 4A). 
356 Based on differential gene expression, we identified these cells as lymphatic endothelial 357 cells (Fig. 4C). Violin plots and gene ontology analysis of Endo Cluster 8 further confirmed 358 the lymphatic endothelial cell fate of this cell cluster (S Fig. 4B,C). In addition to the 359 lymphatic endothelial cell fate commitment genes (PDPN, RELN), Endo Cluster 8 also 360 showed gene expression connected to DNA replication, mitosis and regulation of 361 cytoskeleton organization (CCL21, STMN1, FSCN1, PROX1, TPX2, MAP1B, PLK1,

362 S100A10, NEK2) (S Fig. 4B). These cells showed upregulation of genes associated with 363 inflammation such as PPFIBP1, SEPP1, PTX3, SPHK1 (S Table 6).

364 Interestingly, Endo cluster 2, also identified as a lymphatic endothelial cell cluster, was present in all three sample types, normal myometrium, MED12 variant positive and MED12 variant negative leiomyomas. Endo Cluster 8 was differentiated from Endo Cluster 2

367 by the expression of Nucleolar-spindle-associated protein (NUSAP1), which is an important 368 regulator of mitosis ${ }^{20}$. We confirmed the leiomyoma specific expression of Endo Cluster 8 by 369 immunostaining (Fig. 4E-G). Studies have shown that inflammation is responsible for 370 lymphangiogenesis in different conditions and is responsible for the immune response in 371 these respective conditions ${ }^{21}$. To confirm if the cells in Endo Cluster 8 originated from Endo 372 Cluster 2 in response to the inflammation, we performed pseudotime analysis. Our analysis 373 revealed the expression of Ki67, a proliferation marker in Endo Cluster 8 (S.Fig. 5A,B).

374 Therefore, these data indicate that these cells may have originated from Endo Cluster 2 in 375 leiomyomas in response to the inflammation.

\section{Immune cell infiltration differs by the genotype of leiomyomas}

378 Next, we wanted to analyze the immune profile of the normal myometrium and compare it to 379 the leiomyomas. Our data showed the presence of two subsets of natural killer (NK) cells, B380 cells, dendritic cells, T-cells, CD8+ve T-cells, CD14 monocytes, FCER3A+ monocytes, and 381 two subsets of macrophages in the normal myometrium (Fig. 5A-C). These results are in 382 agreement with the previously reported immune cell populations in myometrium ${ }^{22}$. Missing 
383 cell types in our data that were previously reported to be present in the myometrium are

384 mast cells and neutrophils ${ }^{22-24}$.

385 We found an increase in infiltration of T-cells in the MED12 variant positive 386 leiomyomas (Fig. 5D, H-J). These cells express cytokines and chemoattractant for the 387 recruitment of CD8+ T-cells which have increased cytotoxic activity, indicated by 388 upregulation of GNLY, RHOB, and genes associated with the antigen recognition (TRBV7-9, 389 TRBV9, TRAV38-2DV, TYROBP) (S Fig. 6C; S Table 7). GO enrichment analysis for the 390 biological processes showed upregulation of genes associated with the adaptive immune 391 response and regulation of the immune response (S Fig. 6A). We also found expansion of 392 NK cells expressing CCL21 and CD96 in the MED12 variant positive leiomyomas. These 393 cells showed upregulation of IL-6 secretion, a proinflammatory cytokine and genes 394 responsible for the regulation of the inflammatory response to the antigen stimulation (S 395 Table. 7). Expansion of T-cells, NK cells, and dendritic cells which participate in the adaptive 396 immune response and lymphatic endothelial cells in the MED12 variant positive leiomyomas 397 further suggest that activation of the adaptive immune response plays a role in the 398 management of the MED12 variant positive leiomyomas.

399 In contrast, we observed expansion of macrophages and B-cells in the MED12 400 variant negative leiomyomas (Fig. 5K-M). Macrophages were enriched for ontology terms 401 relevant to regulation of B-cell differentiation and regulation of angiogenesis in addition to 402 known macrophage-functions such as antigen processing (S Fig. 6B). B-cells showed 403 upregulation of the genes IGLV3-11, IGLV3-21, and FCER1G1 which are responsible for 404 antigen recognition and affinity maturation (S Fig. 6D, S Table 7). Tissue immunostaining 405 confirmed the expansion of macrophages and B-cells in the MED12 variant negative 406 leiomyomas compared to MED12 variant positive leiomyomas and normal myometrium (Fig. $4075 \mathrm{D}, \mathrm{K}-\mathrm{M}$, S Fig. 6E-G). These data highlight that immune cell infiltration and the immune 408 response to leiomyomas is dependent on the genotype. 
411 In normal female cells, monoallelic expression of X-chromosome genes is observed due to

412 random inactivation of one of the two X-chromosomes for dosage compensation ${ }^{25}$. This

413 principal is widely used to study the clonality in tumors ${ }^{26}$. Based on this phenomenon,

414 studies utilizing random inactivation of $\mathrm{X}$-linked gene human androgen receptor (HUMARA)

415 and inactive glycose-6-phosphate dehydrogenase (G6PD) isoform expression have

416 suggested that uterine leiomyomas are monoclonal tumors of smooth muscle cells of the

417 uterus ${ }^{27,28}$. However, our single cell sequencing data indicates the presence of cellular

418 heterogeneity in the smooth muscle cell populations, fibroblast populations, and endothelial

419 cell populations in the leiomyomas (Fig. 1B, C). Moreover, MED12 is located on the X-

420 chromosome and is subject to X-chromosome inactivation. We hypothesized that if

421 leiomyomas are truly monoclonal and derive from a so called "stem cell", then different cell

422 types identified in leiomyomas should only express the MED12 mutant variant. Single cell

423 RNA sequencing has an advantage over the previously used techniques to determine the

424 clonality of the tumor because of the ability to capture variants in individual cells and 425 interrogate at higher resolution.

426 We therefore performed variant analysis of the single-cell sequencing datasets of

427 MED12 variant positive leiomyomas using Integrative Genomics Viewer (IGV). IGV analysis

428 revealed expression of both the mutant variants (G44T, G44C) and the wild type MED12

429 allele in the MED12 variant positive leiomyomas (Fig. 6A). To determine which cells express

430 MED12 variant, we generated a UMAP showing smooth muscle cells, fibroblasts and

431 endothelial cells from three MED12 positive fibroid samples (Fig. 6B). We generated the

432 UMAP from only these cells to determine if expression of wild type MED12 is a result of

433 immune cell infiltration in the tumor. We then identified the MED12 variants in the single-cell

434 RNA sequencing data by utilizing the dsc-pileup software developed from previously

435 published tool, demuxlet ${ }^{15,16}$. We plotted the MED12 variants in the UMAP generated using

436 the smooth muscle cell, fibroblast, and endothelial cell populations of the MED12

437 leiomyomas. We found expression of MED12 variants (MED12 G>N - 40\%, MED12 wild

438 type allele- 60\%) in all three cell populations (Fig. 6C). The expression of the MED12 variant 
439 allele and wild type allele varies from patient to patient with some patients expressing

440 roughly $80 \%$ of the MED12 variant allele while others expressed only $20 \%$ of the MED12

441 variant allele (Fig. 6A). These results indicate that MED12 variants present in a limited

442 number of myometrium cells might be sufficient to cause leiomyomas. Upon plotting the wild

443 type MED12 allele of these cells, we found the presence of the wild type MED12 in all

444 clusters of smooth muscle cells, fibroblasts and endothelial cell (Fig. 6C). For the first time,

445 our data indicates that MED12 variant positive leiomyomas might not be monoclonal in 446 nature.

Discussion

449 Uterine leiomyomas are thought to originate from SMC in myometrium. However, 450 recent studies have reported presence of fibroblasts and endothelial cells in uterine 451 leiomyomas in addition to SMC populations ${ }^{10,12}$, thus suggesting presence of cellular 452 heterogeneity in leiomyomas. We applied single cell RNA sequencing technique to create a 453 cell atlas for the normal myometrium and the leiomyomas. Our data shows that at least 18 454 different cell types compose myometrium, including different subtypes of smooth muscle 455 cells, fibroblast, vascular endothelial cells, lymphatic endothelial cells and immune cells.

456 Other than novel lymphatic endothelial cell population exclusive to leiomyomas, myometrium 457 and leiomyomas share similar cell composition. It is therefore likely that transcriptomic 458 differences account for leiomyomagenesis.

459 Previous studies have established the differences in the transcriptomic profile 460 between the normal myometrium and leiomyomas ${ }^{29,30}$. These studies have consistently 461 identified differential expression of genes regulating ECM metabolism (COL12A1, COL6A3, 462 FN1, ADAM19), hormone responsiveness (PRLHR, EGFR, CYP26A1, EGR1), and muscle 463 physiology (RGS2, CACNA1C, MYH2) ${ }^{29,30}$. We analyzed the transcriptional changes in 464 leiomyomas and myometrium at the single cell resolution. Similar to the previous reports we 465 found upregulation of genes involved in ECM metabolism (COL7A1, COL6A2, COL16A1), 466 hormone responsive genes (PTN, FOSB, IGFBP2, FOS), and muscle organization and 
467 differentiation (TTN, LMNA, RGS2) in leiomyomas compared to the normal myometrium. We

468 also examined the effect of underlying leiomyoma genotype on transcriptomic changes.

469 Previous studies have shown that missense variants in MED12 exon 2 associate with 70\%

470 of leiomyomas ${ }^{4-6}$. We compared the transcriptomic changes at single-cell level among

471 MED12 variant positive and MED12 variant negative leiomyomas against the myometrium.

472 We found that both SMC and fibroblast cells show upregulation of genes associated with

473 ECM metabolism in MED12 variant positive leiomyomas, while in MED12 variant negative

474 leiomyomas, only SMC showed such upregulation. Our results are consistent with previous

475 report that both SMC and fibroblasts are responsible for ECM production in MED12 variant

476 positive leiomyomas ${ }^{10}$. Although ECM accumulation is a characteristic feature of

477 leiomyomas there is strikingly increased ECM accumulation in MED12 variant positive

478 leiomyomas compared to MED12 variant negative leiomyomas ${ }^{10}$. It is possible that these

479 transcriptional differences explain increased accumulation of ECM in MED12 variant positive

480 leiomyomas compared to MED12 variant negative leiomyomas.

481 Previous studies using human tissue and transgenic mouse models have implicated

482 the role of $\mathrm{Wnt} / \beta$-catenin, REST-GPR-10, and mTOR signaling pathways in leiomyoma

483 pathology ${ }^{29,31-34}$. In agreement to these studies, our human scRNA seq data here shows

484 dysregulation of WNT signaling, retinoic acid, PI3K, and JAK-STAT signaling pathways in

485 different cell clusters of SMC and fibroblast populations in both MED12 variant positive and

486 MED12 variant negative leiomyomas. However, we found an individual SMC or fibroblast

487 cell cluster can have dysregulation of multiple signaling pathways in both MED12 variant

488 positive and MED12 variant negative leiomyomas. These results indicate that a specific

489 signaling pathway might not be responsible for leiomyoma formation. We have previously

490 shown that common nonsynonymous MED12 exon 2 variant (c.131G>A) causes

491 leiomyomagenesis in a mouse model ${ }^{19}$. Using our MED12 mouse model, we found

492 dysregulation of Wnt signaling pathway, Ras signaling, and mTOR signaling pathways in

493 leiomyomas ${ }^{19}$ The involvement of multiple pathways is likely due to MED12, which plays a

494 known role as part of the mediator complex that regulates global RNA polymerase II 
495 dependent transcription and MED12 variant is likely to have global effect ${ }^{5}$. MED12 variant

496 negative leiomyomas have a highly heterogenous genetic landscape and many of these

497 leiomyomas carry HMGA2 rearrangements ${ }^{5,7}$. HMGA2 belongs to a non-histone

498 chromosomal high-mobility group (HMG) protein family and acts as a transcriptional

499 regulating factor associated with multiple pathologies ${ }^{35,36}$.

$500 \quad$ Uterine leiomyomas are hormone responsive in nature ${ }^{37}$. Previous studies have

501 shown that leiomyomas increase in size with the presence of estrogen and

502 progesterone ${ }^{11,19}$. Leiomyomas with HMGA2 rearrangements and MED12 mutations show

503 increased proliferation of SMC in the presence of estrogen and progesterone, whereas

504 fibroblast population proliferates solely in response to estrogen ${ }^{10}$. Our study shows that all

505 SMC clusters in MED12 variant positive and MED12 variant negative leiomyomas have

506 upregulation of both estrogen and progesterone responsive genes However, we did not

507 observe an enrichment of estrogen or progesterone responsive genes in leiomyoma

508 fibroblast cell clusters (Fig 2I,J, 3M,N, S Table 4,5). Our results indicate that SMCs may be

509 more responsive to ovarian hormones than fibroblasts. It remains to be studied if the

510 fibroblast cells carrying MED12 variant behave like SMC and show increased

511 responsiveness to ovarian hormones or if they interact with SMC in paracrine fashion.

512 Lymphatic endothelial cells were previously reported in myometrium but not in

513 leiomyomas ${ }^{38,39}$. In this study, we found that while normal myometrium has only one

514 lymphatic endothelial cell population, there are two distinct populations of lymphatic

515 endothelial cells in both MED12 variant positive and MED12 variant negative leiomyomas.

516 The lymphatic endothelial cells are known to facilitate the recruitment of immune cells to

517 tissues $^{40}$. Previous studies have reported variable predominance of T-cells and B-cells in

518 leiomyomas ${ }^{41}$. Unexpectedly, we found that the differences in immune cell infiltration in

519 leiomyomas are genotype dependent. While the MED12 variant positive leiomyomas

520 showed increased infiltration of T-cells and NK cells. MED12 variant negative leiomyoma

521 show predominance of B-cells and macrophages suggesting activation of humoral immune

522 response. The increased infiltration of the immune cells in leiomyomas compared to the 
523 normal myometrium can be explained by the presence of additional lymphatic endothelial

524 cells. Why the immune cell infiltration in the leiomyomas differs with respect to their

525 genotype and functional significance needs to be explored in future studies. Collectively,

526 these findings demonstrate underlying cellular heterogeneity in both leiomyomas and

527 myometrium. Our data also explains that previously reported immune cell population

528 infiltration variability in leiomyomas is genotype dependent.

529 Based on studies utilizing HUMARA assay and inactivation of glucose-6-phosphate

530 dehydrogenase isoform expression, uterine leiomyomas are widely accepted to be

531 monoclonal in nature ${ }^{27,28}$. These assays are widely used to study the clonality in different

532 tumors and tissues ${ }^{42}$. However, recent studies have questioned the accuracy of these

533 assays partly affected by inconsistent methylation of $\mathrm{X}$-chromosome genes ${ }^{43,44}$. MED12

534 mutation is responsible for leiomyoma formation in $70 \%$ of women ${ }^{4}$. MED12 is located on the

535 X-chromosome and because of $\mathrm{X}$ chromosome inactivation either the variant or the wild type

536 MED12 allele will be expressedl ${ }^{45}$. It has previously been shown that MED12 variant positive

537 leiomyomas express the variant allele ${ }^{4,5}$. If leiomyomas were monoclonal and originated

538 from a single MED12 variant carrying stem cell, then all of the cells present in a leiomyoma

539 would be expected to carry the same MED12 variant. Our analysis of the data, exploiting the

540 unique genotype of MED12 variant positive leiomyomas, shows that uterine leiomyomas

541 cellular moiety is not monoclonal in nature. We found that MED12 variant positive

542 leiomyomas were composed of a mixture of cells expressing both MED12 variant allele as

543 well as MED12 wild type allele. Other studies using alternative techniques support our

544 findings. FACS sorting of leiomyomas into SMC and fibroblast populations revealed that

545 MED12 variant positive leiomyomas are composed of SMC carrying MED12 variant allele

546 while the fibroblasts cells in leiomyomas do not carry the MED12 variant ${ }^{10}$. The study

547 concluded that the leiomyoma causative mutations are present in SMC only. We, however,

548 found that leiomyomas are a mixture of cells expressing both MED12 variant and wild type

549 allele in multiple cell types examined including SMC, and endothelial cells. Although our 
550 results show that leiomyoma cellular moiety is not monoclonal, the origin of leiomyomas

551 remains uncertain.

552 In conclusion, the single cell atlas of human myometrium and leiomyomas show

553 cellular heterogeneity and complexity that is genotype dependent. The main differences

554 include novel, leiomyoma specific lymphatic endothelial cell type, genotype dependent

555 immune infiltration and transcriptomic changes that may account for hormone

556 responsiveness and ECM accumulation. Moreover, our studies show that leiomyoma cell

557 moiety is not monoclonal in nature, which should be a major consideration when designing

558 future therapeutics against leiomyomas.

559 Code availability

560 The datasets generated and analyzed in the study are available in the NCBI Gene

561 Expression Omnibus (GEO) and Sequence Read Archive (SRA) and can be accessed upon

562 request. All custom scripts can be accessed upon request to the Lead Contacts.

\section{Contributions}

564 JG and AR conceived the study, JG designed and performed the experiments, data

565 analysis and interpretation. AR supervised the study, designed experiments, and

566 performed data analysis. JR performed computational analysis. JJW helped with

567 the pathological examination of the tissues used in the study. S.B and D.C helped with data

568 analysis. JG and AR wrote the manuscript with input from all the authors.

\section{Acknowledgments}

570 We would like to thank Dr. Rohit K. Gupta for helping us with the sample preparation

571 protocol. Meghana Sukhthankar for helping with the sample preparation. Dr. Katja Rust and

572 Tania Moody for discussions about the data analysis. Matthew Tae for help with GO

573 analysis. We would like to thank all the Rajkovic lab members for the critical reading of the 
574 document. This work was supported by funding from National Institute of Child Health and

575 Human Development (5P50HD098580).

\section{Conflict of interest}

577 The authors declare no conflict of interest.

\section{References}

579 1. Stewart, E.A. Uterine fibroids. Lancet 357, 293-298 (2001).

580 2. Cardozo, E.R., et al. The estimated annual cost of uterine leiomyomata in the United

$581 \quad$ States. Am J Obstet Gynecol 206, 211.e211-219 (2012).

582 3. Farquhar, C.M. \& Steiner, C.A. Hysterectomy rates in the United States 1990-1997.

$583 \quad$ Obstet Gynecol 99, 229-234 (2002).

584 4. McGuire, M.M., et al. Whole exome sequencing in a random sample of North

585 American women with leiomyomas identifies MED12 mutations in majority of

$586 \quad$ uterine leiomyomas. PLoS One 7, e33251 (2012).

587 5. Mäkinen, N., et al. MED12, the mediator complex subunit 12 gene, is mutated at

588 high frequency in uterine leiomyomas. Science 334, 252-255 (2011).

589 6. Halder, S.K., et al. Novel MED12 gene somatic mutations in women from the

590 Southern United States with symptomatic uterine fibroids. Mol Genet Genomics 290,

$591 \quad 505-511$ (2015).

592 7. Yatsenko, S.A., et al. Highly heterogeneous genomic landscape of uterine

593

594

595

596

597

598

599

600

601

602

603

604

605

606

607

608

609

610

611

612

613

614 leiomyomas by whole exome sequencing and genome-wide arrays. Fertil Steril 107, 457-466 e459 (2017).

8. Mehine, M., et al. Characterization of uterine leiomyomas by whole-genome sequencing. N Engl J Med 369, 43-53 (2013).

9. Rein, M.S., et al. Cytogenetic abnormalities in uterine myomas are associated with myoma size. Mol Hum Reprod 4, 83-86 (1998).

10. Wu, X., et al. Subtype-Specific Tumor-Associated Fibroblasts Contribute to the

11. Bulun, S.E. Uterine fibroids. N Engl J Med 369, 1344-1355 (2013).

12. Holdsworth-Carson, S.J., Zaitseva, M., Girling, J.E., Vollenhoven, B.J. \& Rogers, P.A. Common fibroid-associated genes are differentially expressed in phenotypically dissimilar cell populations isolated from within human fibroids and myometrium. Reproduction 147, 683-692 (2014).

13. Goad, J., Ko, Y.A., Kumar, M., Syed, S.M. \& Tanwar, P.S. Differential Wnt signaling activity limits epithelial gland development to the anti-mesometrial side of the mouse uterus. Dev Biol 423, 138-151 (2017).

14. Stuart, T., et al. Comprehensive Integration of Single-Cell Data. Cell 177, 18881902.e1821 (2019).

15. Pique-Regi, R., et al. Single cell transcriptional signatures of the human placenta in term and preterm parturition. Elife 8(2019).

16. Kang, H.M., et al. Multiplexed droplet single-cell RNA-sequencing using natural genetic variation. Nat Biotechnol 36, 89-94 (2018). 
615

616

617

618

619

620

621

622

623

624

625

626

627

628

629

630

631

632

633

634

635

636

637

638

639

640

641

642

643

644

645

646

647

648

649

650

651

652

653

654

655

656

657

658

659

660

17. Latif, N., Sarathchandra, P., Chester, A.H. \& Yacoub, M.H. Expression of smooth muscle cell markers and co-activators in calcified aortic valves. Eur Heart J 36, 13351345 (2015).

18. Holdsworth-Carson, S.J., Zaitseva, M., Vollenhoven, B.J. \& Rogers, P.A. Clonality of smooth muscle and fibroblast cell populations isolated from human fibroid and myometrial tissues. Mol Hum Reprod 20, 250-259 (2014).

19. Mittal, P., et al. Med12 gain-of-function mutation causes leiomyomas and genomic instability. J Clin Invest 125, 3280-3284 (2015).

20. Iyer, J., Moghe, S., Furukawa, M. \& Tsai, M.Y. What's Nu(SAP) in mitosis and cancer? Cell Signal 23, 991-998 (2011).

21. Kim, H., Kataru, R.P. \& Koh, G.Y. Inflammation-associated lymphangiogenesis: a double-edged sword? J Clin Invest 124, 936-942 (2014).

22. Siewiera, J. \& Erlebacher, A. Myometrial leukocytes. Current Opinion in Physiology 13, 6-13 (2020).

23. FOX, J.E. \& ABELL, M.R. MAST CELLS IN UTERINE MYOMETRIUM AND LEIOMYOMATOUS NEOPLASMS. Am J Obstet Gynecol 91, 413-418 (1965).

24. Thomson, A.J., et al. Leukocytes infiltrate the myometrium during human parturition: further evidence that labour is an inflammatory process. Hum Reprod 14, 229-236 (1999).

25. Harper, P.S. Mary Lyon and the hypothesis of random X chromosome inactivation. Hum Genet 130, 169-174 (2011).

26. Chen, G.L. \& Prchal, J.T. X-linked clonality testing: interpretation and limitations. Blood 110, 1411-1419 (2007).

27. Mashal, R.D., et al. Analysis of androgen receptor DNA reveals the independent clonal origins of uterine leiomyomata and the secondary nature of cytogenetic aberrations in the development of leiomyomata. Genes Chromosomes Cancer 11, 1-6 (1994).

28. Linder, D. \& Gartler, S.M. Glucose-6-phosphate dehydrogenase mosaicism: utilization as a cell marker in the study of leiomyomas. Science 150, 67-69 (1965).

29. Mehine, M., et al. Integrated data analysis reveals uterine leiomyoma subtypes with distinct driver pathways and biomarkers. Proc Natl Acad Sci U S A 113, 1315-1320 (2016).

30. Moyo, M.B., Parker, J.B. \& Chakravarti, D. Altered chromatin landscape and enhancer engagement underlie transcriptional dysregulation in MED12 mutant uterine leiomyomas. Nat Commun 11, 1019 (2020).

31. Tanwar, P.S., et al. Constitutive activation of Beta-catenin in uterine stroma and smooth muscle leads to the development of mesenchymal tumors in mice. Biol Reprod 81, 545-552 (2009).

32. Varghese, B.V., et al. Loss of the repressor REST in uterine fibroids promotes aberrant $\mathrm{G}$ protein-coupled receptor 10 expression and activates mammalian target of rapamycin pathway. P Natl Acad Sci USA 110, 2187-2192 (2013).

33. Commandeur, A.E., Styer, A.K. \& Teixeira, J.M. Epidemiological and genetic clues for molecular mechanisms involved in uterine leiomyoma development and growth. Hum Reprod Update 21, 593-615 (2015).

34. Prizant, H., et al. Uterine-specific loss of Tsc2 leads to myometrial tumors in both the uterus and lungs. Mol Endocrinol 27, 1403-1414 (2013). 
661

662

663

664

665

666

667

668

669

670

671

672

673

674

675

676

677

678

679

680

681

682

683

684

685

686

687

688

689

690

691

692
35. Fusco, A. \& Fedele, M. Roles of HMGA proteins in cancer. Nat Rev Cancer 7, 899-910 (2007).

36. Pfannkuche, K., Summer, H., Li, O., Hescheler, J. \& Droge, P. The high mobility group protein HMGA2: a co-regulator of chromatin structure and pluripotency in stem cells? Stem Cell Rev Rep 5, 224-230 (2009).

37. Kim, J.J. \& Sefton, E.C. The role of progesterone signaling in the pathogenesis of uterine leiomyoma. Mol Cell Endocrinol 358, 223-231 (2012).

38. Koukourakis, M.I., et al. LYVE-1 immunohistochemical assessment of lymphangiogenesis in endometrial and lung cancer. J Clin Pathol 58, 202-206 (2005).

39. Red-Horse, K. Lymphatic vessel dynamics in the uterine wall. Placenta 29 Suppl A, S55-59 (2008).

40. Lucas, E.D. \& Tamburini, B.A.J. Lymph Node Lymphatic Endothelial Cell Expansion and Contraction and the Programming of the Immune Response. Front Immunol 10, 36 (2019).

41. Crow, J. Pathology of uterine fibroids. Baillieres Clin Obstet Gynaecol 12, 197-211 (1998).

42. Kopp, P., et al. Clonal X-inactivation analysis of human tumours using the human androgen receptor gene (HUMARA) polymorphism: a non-radioactive and semiquantitative strategy applicable to fresh and archival tissue. Mol Cell Probes 11, 217-228 (1997).

43. Swierczek, S.I., et al. Methylation of AR locus does not always reflect X chromosome inactivation state. Blood 119, e100-109 (2012).

44. Carrel, L. \& Willard, H.F. X-inactivation profile reveals extensive variability in X-linked gene expression in females. Nature 434, 400-404 (2005).

45. Ercan, S. Mechanisms of $x$ chromosome dosage compensation. J Genomics 3, 1-19 (2015). 

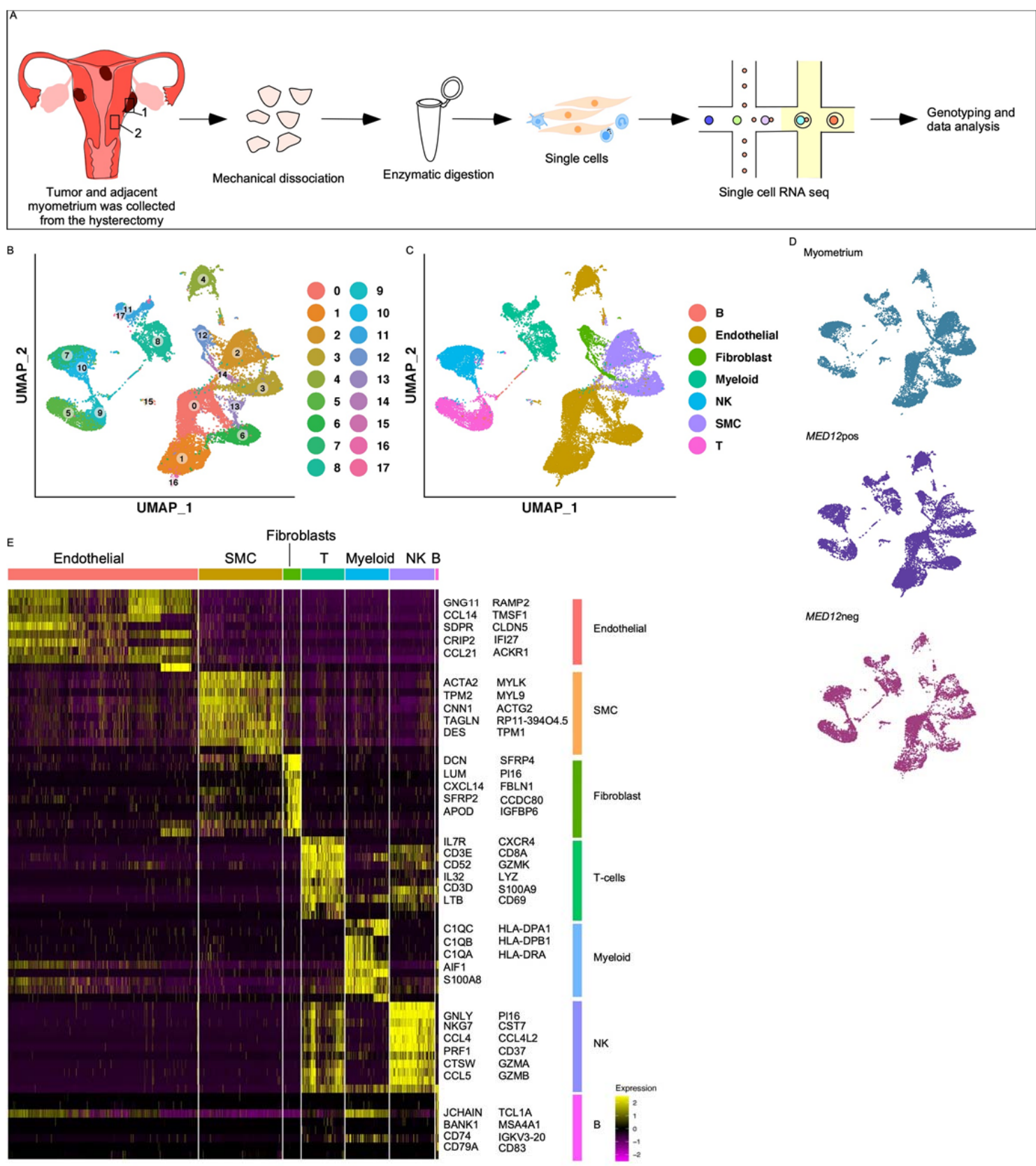

MED12neg

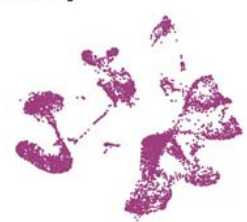

694

695 Fig.1: Single cell atlas of normal myometrium and leiomyomas. A) Overview of the

696 dissociation protocol for both normal myometrium $(n=5)$ and leiomyomas $(n=8)$. B)

697 Clustering of 34,435 high quality cells from MED12 variant positive leiomyomas, MED12 negative leiomyomas and normal myometrium. C) Cell lineages identified by the marker

699 gene expression D) Annotation of the cell clusters per sample. E) Heatmap showing the top

70010 genes used for cluster identification. Columns denote cells, rows denote genes. 


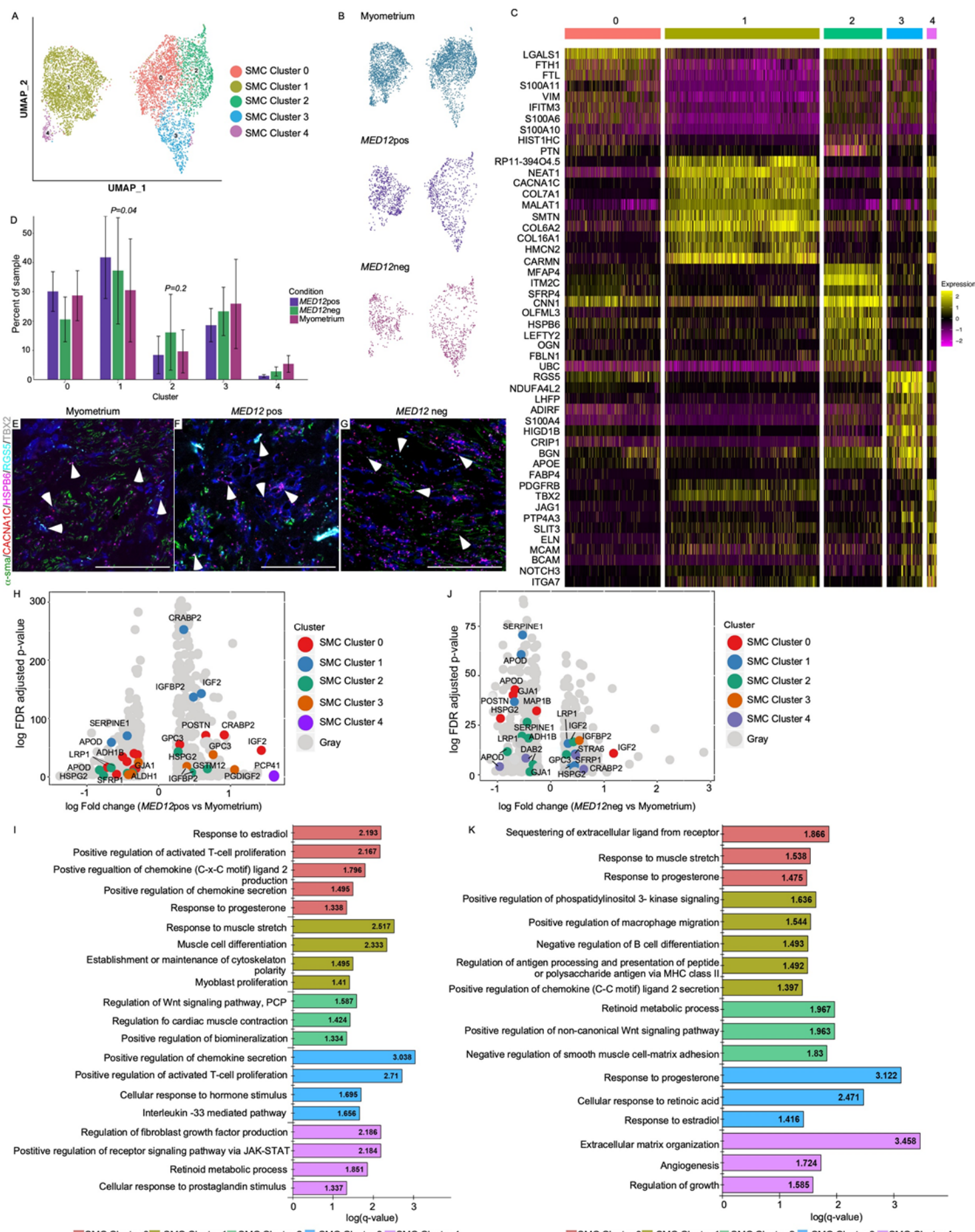

702 Fig.2: Intracellular heterogeneity and transcriptomic changes in the MED12 variant

703 positive and MED12 variant negative smooth muscle cell clusters in comparison to

704 the normal myometrium. A) UMAP showing the clusters of 6500 smooth muscle cells B)

705 UMAP showing that all cell clusters are present in all three conditions- myometrium, MED12

706 variant positive leiomyomas and MED12 variant negative leiomyomas. C) Heatmap of the 
707 smooth muscle cell clusters. Colored bar on the top represents cluster number, Columns

708 denote cells; rows denote genes. D) Cell proportion changes in the MED12 variant positive

709 and MED12 variant negative leiomyomas in comparison to normal myometrium. Data

710 represented here are mean \pm s.e.m. $P$ values were determined by Dirichlet-multinomial

711 regression E-G) in situ images showing the validation of the smooth muscle cell clusters in

712 MED12 variant positive leiomyomas, MED12 variant negative leiomyomas and normal

713 myometrium. Scale bar is $100 \mu \mathrm{m} . \mathrm{H}$ ) Volcano plots showing the transcriptomic changes in

714 the smooth muscle cell clusters of MED12 variant positive compared to the normal

715 myometrium. I) GO analysis of DE genes showing at least 1.5 fold change in the MED12

716 variant positive leiomyomas as compared to the normal myometrium J) Volcano plots

717 showing the transcriptomic changes in the smooth muscle cell clusters of MED12 variant

718 negative compared to the normal myometrium K) GO analysis of DE genes showing at least

7191.5 fold change in the MED12 variant negative leiomyomas as compared to the normal 720 myometrium. 

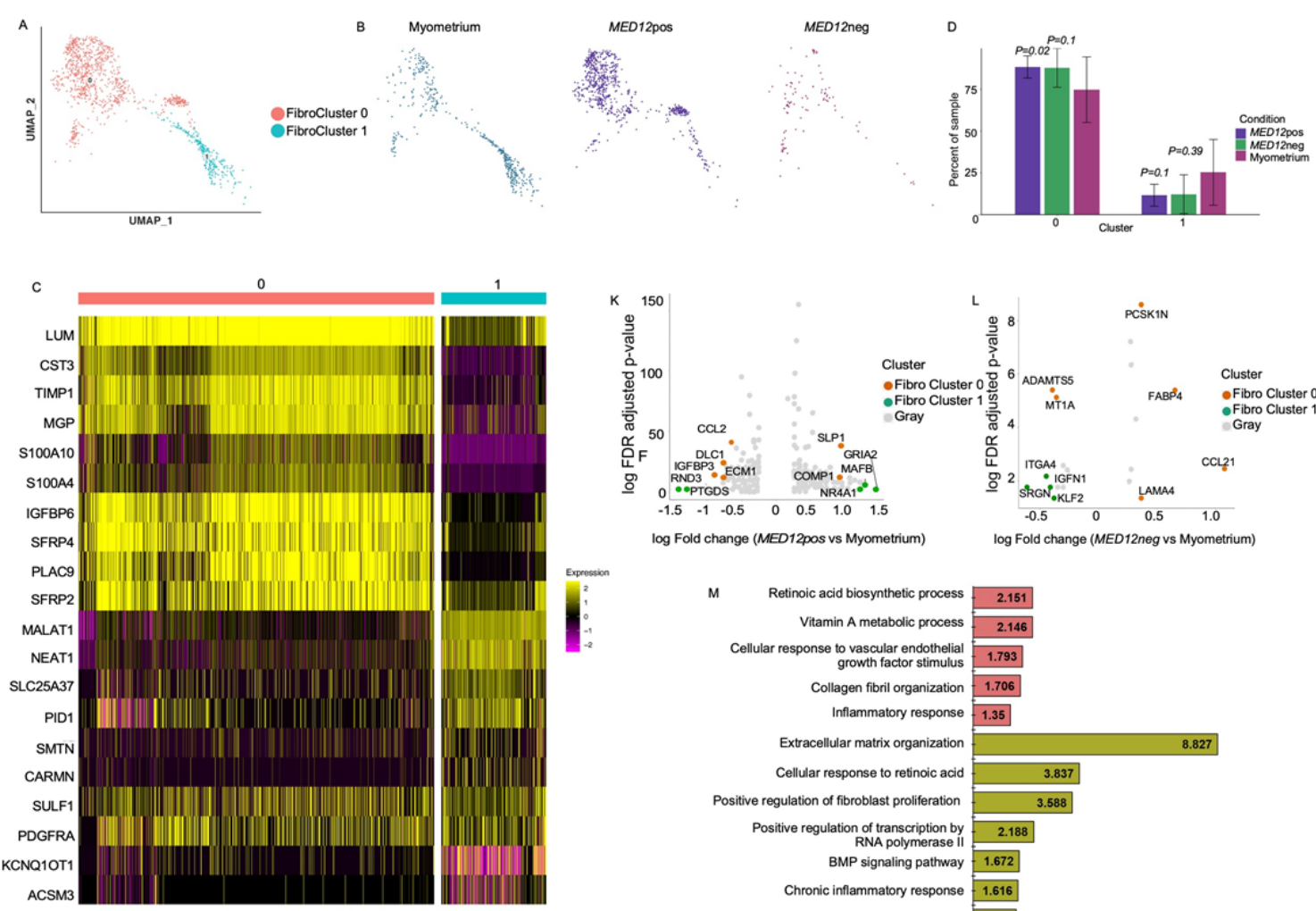

$\log$ Fold change (MED12pos vs Myometrium)
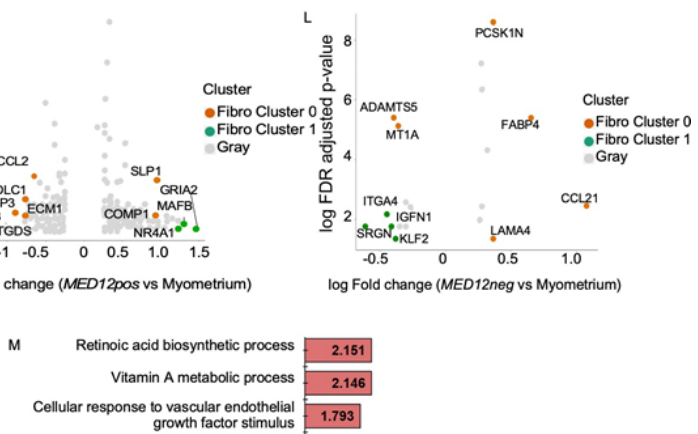

Collagen fibril organization 1.706

Inflammatory response 1.35

Extracellular matrix organization

Cellular response to retinoic acid

Positive regulation of fibroblast proliferation

Positive regulation of transcription by
RNA polymerase II

BMP signaling pathway -1.672

Chronic inflammatory response 1.616
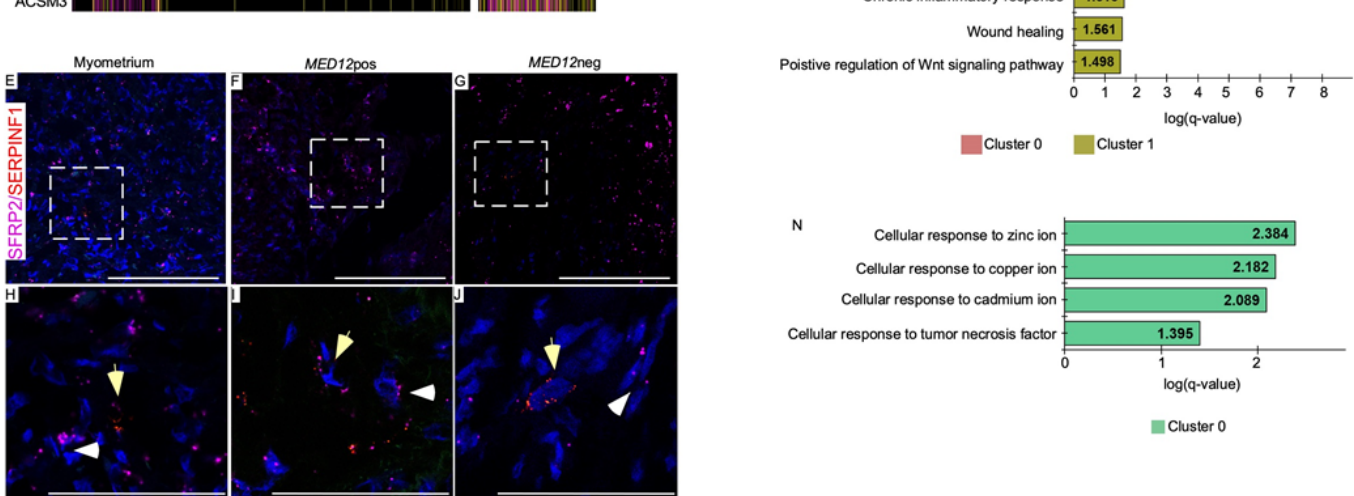

MED12neg

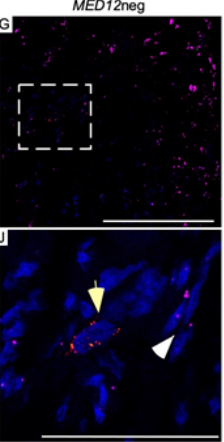

$\log (q$-value $)$

Cluster 0 Cluster 1

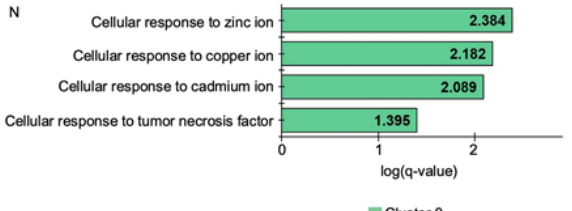

721

Fig.3: Intracellular heterogeneity and transcriptomic changes in the MED12 variant

725 showing that all cell clusters are present in all three conditions- myometrium, MED12 variant

726 positive leiomyomas and MED12 variant negative leiomyomas C) Heatmap of the fibroblast

727 cell clusters. Colored bar on the top represents cluster number, Columns denote cells; rows

728 denote genes. D) Cell proportion changes in the MED12 variant positive and MED12 variant

729 negative leiomyomas in comparison to the normal myometrium. Data represented here are

730 mean \pm s.e.m. $P$ values were determined by Dirichlet-multinomial regression E-J) in-situ 
731 images showing the validation of fibroblast populations SFRP2 (Fibro Cluster 0; white

732 arrowheads), and SERPINF1 (Fibro Cluster 1; yellow arrowheads) in normal myometrium,

733 MED12 variant positive leiomyomas and MED12 variant negative leiomyomas. Scale bar is

$734100 \mu \mathrm{m} . \mathrm{K}$ ) Volcano plots showing the transcriptomic changes in the fibroblast cell clusters

735 of MED12 variant positive compared to the normal myometrium L) Volcano plots showing

736 the transcriptomic changes in the fibroblast cell clusters of MED12 variant negative

737 compared to the normal myometrium M) GO analysis of DE genes showing at least 1.5 fold

738 change in the MED12 variant negative leiomyomas as compared to the normal myometrium.

739 No DE genes were found in cluster 1 in comparison to the normal myometrium. N) GO

740 analysis of DE genes showing at least 1.5 -fold change in the MED12 variant positive

741 leiomyomas as compared to the normal myometrium

742

743

744 

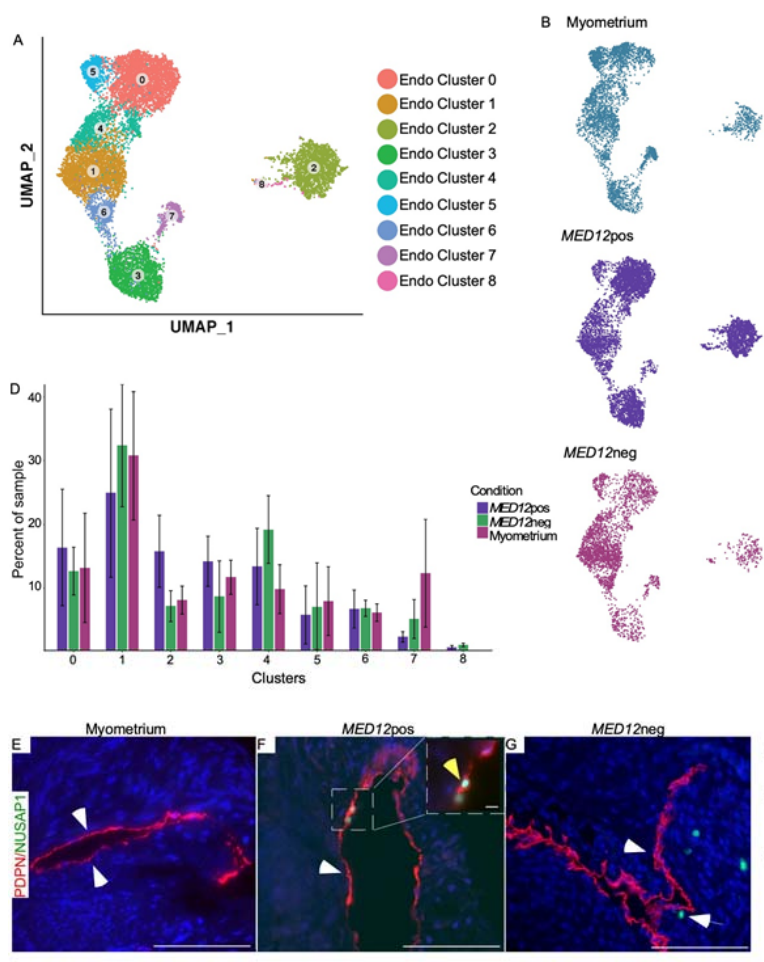

Fig.4: Novel lymphatic endothelial cells are present in leiomyomas A) UMAP showing

747 the clusters of 14742 endothelial cells B) UMAP showing the cluster annotation per

748 condition- myometrium, MED12 variant positive leiomyomas and MED12 variant negative

749 leiomyomas C) Heatmap of endothelial cell clusters. Colored bar on the top represents

750 cluster number, Columns denote cells; rows denote genes. D) Cell proportion changes in the

751 MED12 variant positive and MED12 variant negative leiomyomas in comparison to the

752 normal myometrium. Data represented here are mean \pm s.e.m. $P$ values were determined by

753 Dirichlet-multinomial regression E) Immunostaining for PDPN (white arrowheads) and

754 NUSAP1 (yellow arrowheads) showing presence of lymphatic endothelial cell clusters in

755 MED12 variant positive leiomyomas, MED12 variant negative leiomyomas and normal

756 myometrium. Scale bar is $100 \mathrm{um}$. 
758

759

760

761

762

763

764

765

766

767

768

769

770

771

772
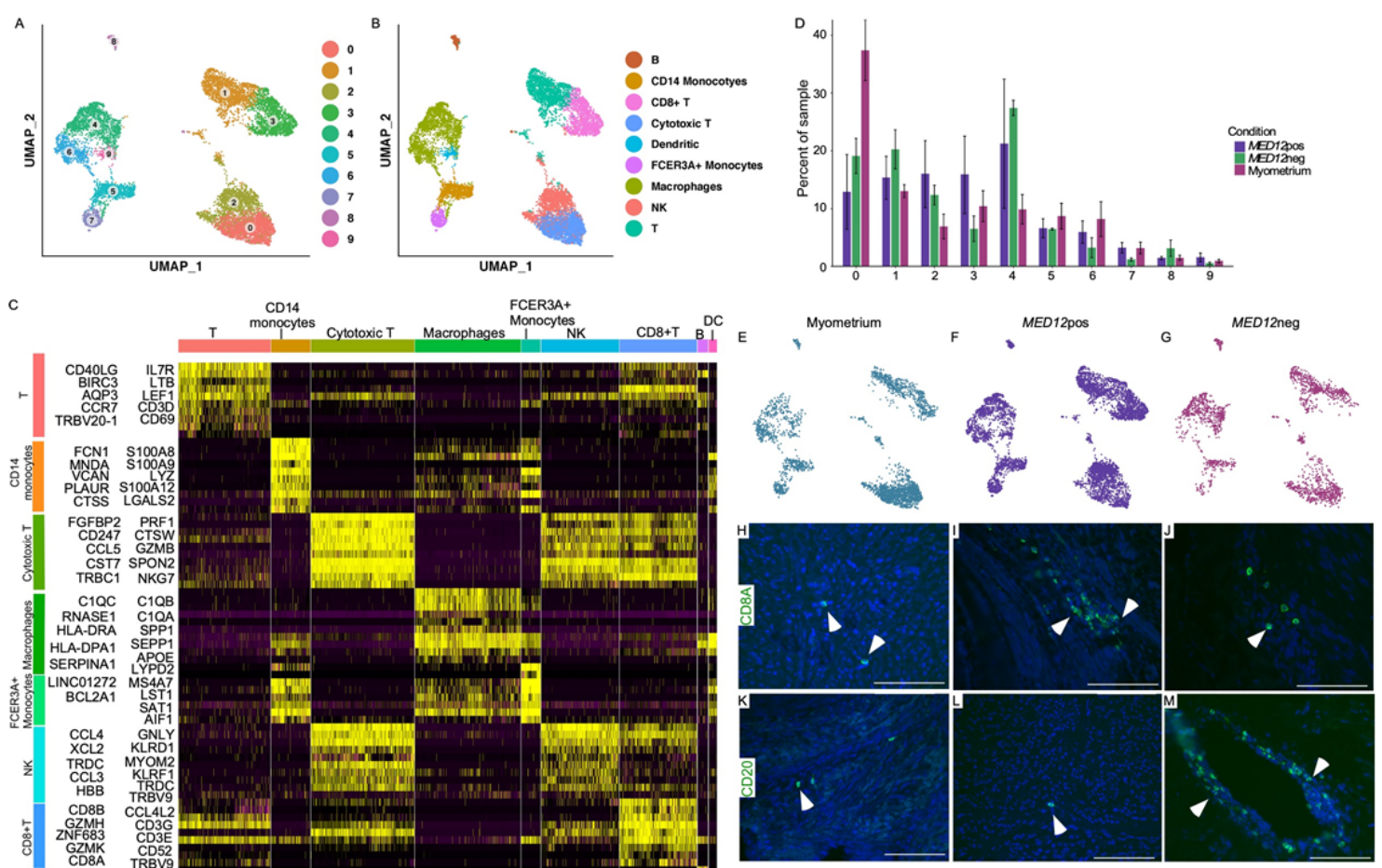

$\mathrm{CD} 37$
$\mathrm{C} 3 \mathrm{~A}$ HLA-DPB1 MSAA1
MLADOA1
HAA-DQB1 FCERTA

Fig.5: Immune cell infiltration is dependent on genotype of the uterine leiomyoma A)

UMAP showing the immune cell clusters. B) Annotation of the UMAP clusters. C) Heatmap of endothelial cell clusters. Colored bar on the top represents cluster number. Columns denote cells; rows denote genes. D) Cell proportion changes in the MED12 variant positive and MED12 variant negative leiomyomas in comparison to the normal myometrium. Data represented here are mean \pm s.e.m. $P$ values were determined by Dirichlet-multinomial regression E-G) UMAP showing the cluster annotation per condition- myometrium, MED12 variant positive leiomyomas and MED12 variant negative leiomyomas respectively. $\mathrm{H}-\mathrm{J}$ ) CD8a (white arrowheads) showing increase infiltration of T-cells-in MED12 variant positive leiomyomas, as compared to normal myometrium and MED12 variant negative leiomyomas. K-M) CD-20 staining (white arrowheads) showing increased infiltration of B-cells in MED12 variant negative leiomyomas compared to normal myometrium and MED12 variant positive leiomyomas. Scale bar is $100 \mathrm{um}$. 
bioRxiv preprint doi: https://doi.org/10.1101/2020.12.21.402313; this version posted December 22, 2020. The copyright holder for this preprint (which was not certified by peer review) is the author/funder, who has granted bioRxiv a license to display the preprint in perpetuity. It is made available under aCC-BY-NC-ND 4.0 International license.

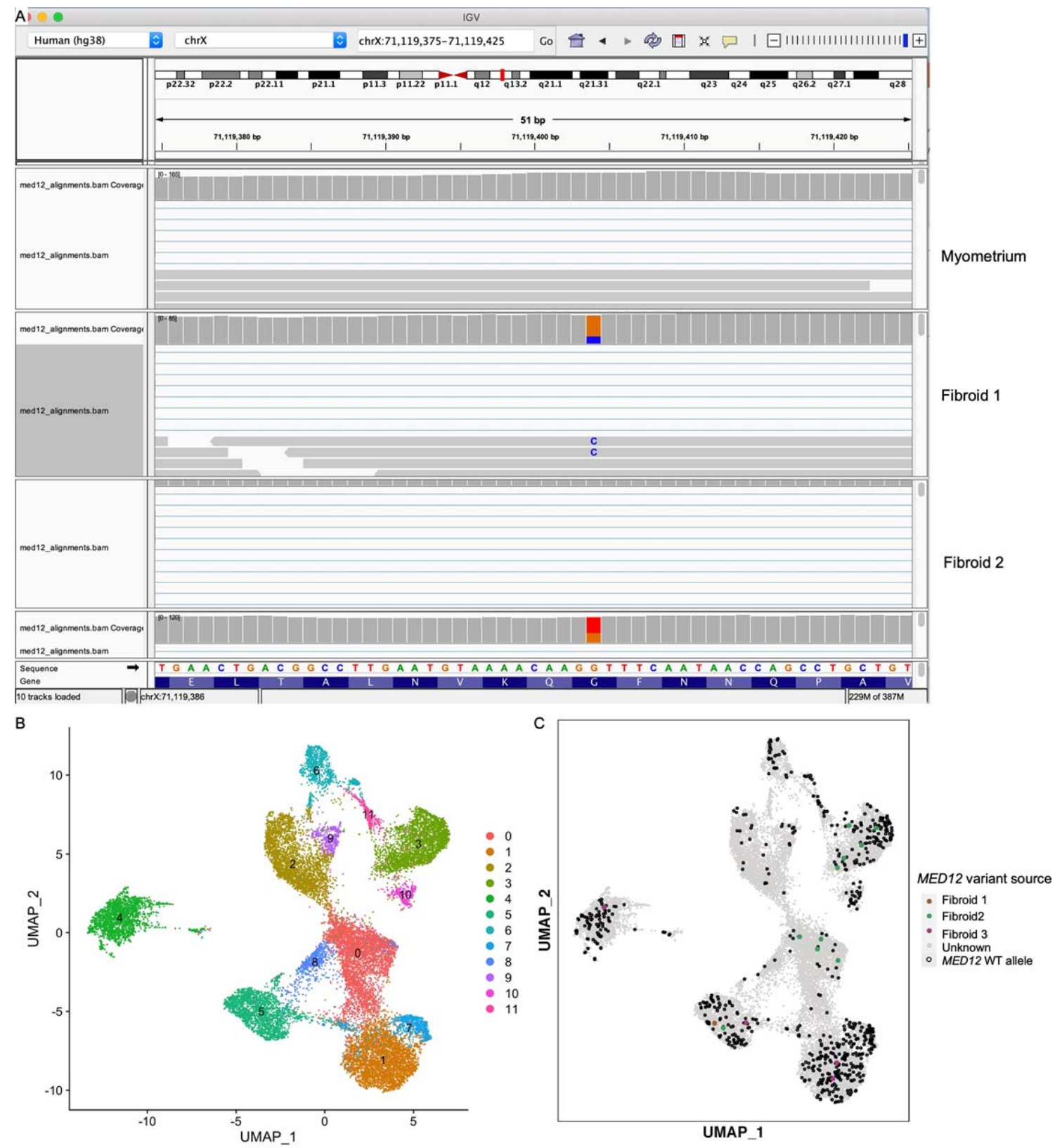

Fig.6: MED12 variant positive uterine leiomyomas are not monoclonal A) IGV analysis

showing presence of both the variant and wild type codon at c131 in the MED12 variant positive leiomyomas. B) UMAP of the mesenchymal cell populations in the leiomyomas.

777 Clusters $0,1,5,7,8$ are endothelial cells, Cluster 2, 3 and 9 are smooth muscle cells, 778 clusters 11 and 6 are fibroblasts, clusters 4 and 10 are lymphatic endothelial cells. C) UMAP 779 showing the presence of the mutant MED12 variant (colored dots) in all smooth muscle cells, fibroblasts and endothelial cells and the wild type MED12 (black dots). 